\title{
Fetal brain disruption sequence in a newborn infant with a history of cordocentesis at 21 weeks gestation
}

\author{
N Villó, J Beceiro, M Cebrero, E Garcia de Frias
}

\begin{abstract}
The case is reported of a full term infant with severe microcephaly, overlapping sutures, prominence of the occipital bone, and scalp rugae. No other associated malformations were observed. The only obstetric history of interest was the performance of cordocentesis at 21 weeks gestational age because of low maternal $\alpha$ fetoprotein levels. Ultrasound scans performed until then were normal. Cranial growth retardation was detected on ultrasound scanning at 25 weeks and intrauterine growth retardation as well as severe microcephaly at 34 weeks. Neuroimaging studies performed on the newborn infant showed intense cerebral atrophy in both hemispheres. Other complementary investigations gave negative results. A relation is proposed between the cordocentesis and the development of vascular disruption, which could have caused the fetal brain disruption sequence in this case. (Arch Dis Child Fetal Neonatal Ed 2001;84:F63-F64)
\end{abstract}

Keywords: cordocentesis; microcephaly; disruption; vascular encephalopathy; brain; cerebral atrophy

The fetal brain disruption sequence comprises a recognisable pattern of birth defects consisting of severe microcephaly, overlapping sutures, prominence of the occipital bone, and scalp rugae. Partial brain necrosis resulting in collapse of the skull and severe microcephaly is postulated to arise after 12 weeks of gestation as a consequence of vascular disruption, viral infection, or hypertermia. Brain development until that time is apparently normal.

Department of Pediatrics, University Hospital Principe de Asturias, Alcalá de

Henares, Madrid,

Spain

N Villó

J Beceiro

M Cebrero

E Garcia de Frias

Correspondence to: Dr Villó, Servicio de Pediatria, Hospita Universitario Príncipe de Asturias, Carretera de Alcalá-Meco s/n, Alcalá de Henares, Madrid, España email:

jbeceiro@hupa.insalvol.es

Accepted 4 May 2000

\section{Clinical report}

A female infant was born at 38 weeks gestation. She was the product of the first pregnancy of unrelated healthy white parents. Her mother was 27 years old. Pregnancy had been uneventful with no known exposure to toxins, radiation, or accidents. There was no history of ingestion of potentially teratogenic drugs, and no episodes of fever or infection were recalled. Low levels of $\alpha$ fetoprotein in the maternal serum were detected at 21 weeks, and cordocentesis was performed apparently without complications and showed a normal karyotype (46XX). The mother explained that the procedure had been laborious and had required multiple punctures and extractions to obtain fetal blood. Ultrasound scans were reported to be normal until 21 weeks gestational age. A

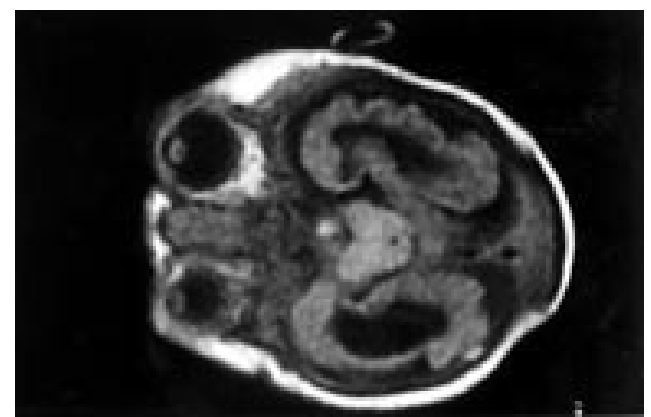

Figure 1 Cerebral magnetic resonance image. Axial section showing a marked decrease in both cerebral hemispheres and ventriculomegaly. There is appreciable parenchymal atrophy, although there is formation of gyri.

slight cranial growth retardation was detected at 25 weeks and intrauterine growth retardation with severe microcephaly after 34 weeks. Spontaneous vaginal delivery ensued at 38 weeks gestational age. The $\mathrm{pH}$ in the cord artery blood was 7.31 and the Apgar score was $8-9$. The newborn infant's weight, length, and occipitofrontal circumference were $2055 \mathrm{~g}$ (< 10th percentile), $46 \mathrm{~cm}$ (10th-25th percentile), and $26 \mathrm{~cm}$ (<3rd percentile) respectively. Physical examination showed microcephaly with intense overlapping of all cranial sutures and prominence of the occipital bone as well as scalp rugae with normal hair pattern. No other malformations were detected. She exhibited normal spontaneous activity and appropriate tone and strength for gestational age. Tendon reflexes were normal, without clonus or tremor. Primitive reflexes, sucking, and swallowing reflexes were present, and heart rate was normal at 30 beats $/ \mathrm{min}$. The response to acoustic and visual stimuli was poor, with inability to fix on objects or to follow them. She was irritable with frequent high pitched crying. A simple cranial $x$ ray photograph showed microcephaly with intense overlapping of all cranial sutures. Cranial computed tomography and brain magnetic resonance imaging showed appreciable cerebral parenchymal atrophy, gyri in both cerebral hemispheres, and ventriculomegalia with an increased subarachnoid space (fig 1). The brainstem, cerebellum, and third and fourth ventricles were normal (fig 2). The ocular fundus showed bilateral optic hypoplasia.

The abdominal ultrasound scan did not show any pathological findings. A serological screen for congenital infection was negative, and the shell vial technique did not detect cytomegalovirus in the urine. A cytochemical examination of cerebrospinal fluid showed it to 


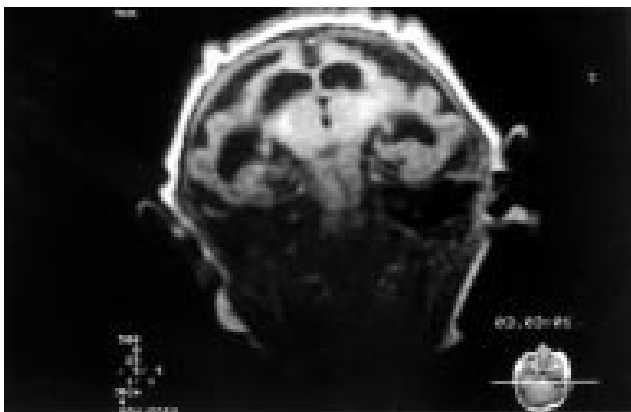

Figure 2 Cerebral magnetic resonance image: coronal section. The brainstem, mesencephalon, and cerebellum are normal.

be normal. Virus isolation in cell cultures of cerebrospinal fluid was negative as well as genomic amplification by polymerase chain reaction for herpes virus. Follow up during the first 3 years of life showed progressively abnormal neurological development with generalised hypertonia, spasticity, and seizures. No other organs or systems were affected during this period.

\section{Discussion}

The clinical and neuroimaging findings for this newborn infant correspond to those reported by Russell et al ${ }^{1}$ which are described as a fetal brain disruption sequence. This sequence of defects occurs in a fetus that has had normal brain growth during the first 12 weeks of gestation, a period in which the basic structures are developed and the normal hair pattern appears. After this period, something damages the fetal brain causing partial necrosis, followed by a decrease in intracranial pressure and collapse of the fetal skull. Three possible causes of this sequence have been suggested: vascular disruption, viral infection, and hypertermia. ${ }^{12} \mathrm{~A}$ possible genetic origin was reported in the case of two sisters who developed clinical findings compatible with an unknown storage disorder. ${ }^{3}$ The negative results of the viral and serological investigations as well as the absence of maternal hypertermia suggest vascular disruption to be the most likely cause in this case. No associated alterations pointing to a genetic origin were observed. Encephalopathies with a vascular aetiology may have a maternal or fetal origin, when there is an interruption of the blood flow or the oxygen supply to the fetal brain, either because of a haemorrhagic or thromboembolic accident or because of a state of shock or severe hypoxia in the mother. ${ }^{4}$ Cases of intrauterine cerebral infarction have been described, especially in the middle cerebral artery zones, secondary to embolism of placental tissue originating in fetal umbilical veins, which could reach the fetal cerebral arterial system because of the right to left shunt present in the fetal circulatory system. ${ }^{56}$ Embolism of necrotic tissue can also occur in the case of the antenatal death of a monozygotic twin, causing cerebral lesions in the surviving twin that vary from porencephalic cyst or multicystic encephalomalacia to hydranencephaly in the most severe cases. ${ }^{78}$ To our knowledge, no case of fetal brain vascular disruption as the result of fetal cordocentesis has been reported. The most common complications reported for cordocentesis are fetal haemorrhage, umbilical cord haematoma, reflex fetal bradycardia, distal chorioamnionitis, and fetomaternal transfusion. ${ }^{9}$ Fetal death directly attributable to this procedure can occur in up to $4.9 \%$ of cases during the two weeks after the procedure, and a high percentage of these are related to chorioamnionitis. In the case we present here, the only hazardous event during pregnancy was the performance of cordocentesis at 21 weeks gestation. Acute alterations in both umbilical and cerebral fetal blood flow induced by cordocentesis have been reported, although the exact mechanism and clinical implications of these findings are not known. ${ }^{10}$ Given the chronological sequence of ultrasound findings during the pregnancy, we believe that vascular disruption during the cordocentesis procedure at 21 weeks gestation could explain the development of the fetal brain disruption sequence in this case. The exact underlying mechanism, however, remains unexplained.

1 Russell LJ, Weaver DD, Bull MJ, Weinbaum M. In utero brain destruction resulting in collapse of the fetal skull, microcephaly, scalp rugae and neurological impairment:
the fetal brain disruption sequence. Am f Med Genet the fetal brain

2 Moore CA, Weaver DD, Bull MJ. Fetal brain disruption sequence. F Pediatr 1990;116:383-6.

3 Alexander IE, Tauro GP, Bankier A. Fetal brain disruption sequence in sisters. Eur F Pediatr 1995;154:654-7.

4 Larroche JC. Fetal encephalopathies of circulatory origin. Biol Neonate 1986;50:61-74.

5 Clark RM, Linell EA. Case report: prenatal occlusion of the internal carotid artery. $\mathcal{f}$ Neurol Neurosurg Psychiat 1954;17:295-7.

6 Cocker J, George SW, Yates PO. Perinatal occlusion of the middle cerebral artery. Dev Med Child Neurol 1965;7:2957 .

7 Hughes HE, Miskin M. Congenital microcephaly due to vascular disruption: in utero documentation. Pediatrics 1986;78:85-7.

8 Yoshiota H, Kadamoto Y, Mino M, Morikawa Y, Kasubuchi Y, Kasunoki T. Multicystic encephalomalacia in liveborn twin with a stillborn macerated co-twin. $\mathcal{F}$ Pediatr 1979;95:798-800.

9 Ghidini A, SepulvedaW, Lockwood CJ, Romero R. Complications of fetal blood sampling. Am $f$ Obstet Gynecol 1993; 168:1339-43.

10 Hecher K, Stettner H, Spernol R, Harrington K, Skay S. The effect of cordocentesis on umbilical and middle cerebral artery flow velocity waveforms. Br F Obstet Gynecol 1993;100:823-31. 